The Astrophysical Journal, 482:83-88, 1997 June 10

(C) 1997. The American Astronomical Society. All rights reserved. Printed in U.S.A.

\title{
DETECTION RATES FOR CLOSE BINARIES VIA MICROLENSING
}

\author{
B. ScOtT GAUdi AND ANDRew Gould ${ }^{1}$ \\ Department of Astronomy, Ohio State University, Columbus, OH 43210; gaudi@payne.mps.ohio-state.edu, \\ gould@payne.mps.ohio-state.edu \\ Received 1996 June 21; accepted 1997 January 2
}

\begin{abstract}
Microlensing is one of the most promising methods of reconstructing the stellar mass function down to masses even below the hydrogen-burning limit. The fundamental limit to this technique is the presence of unresolved binaries, which can, in principle, significantly alter the inferred mass function. Here we quantify the fraction of binaries that can be detected using microlensing, considering specifically the mass ratio and separation of the binary. We find that almost all binary systems with separations greater than $b \sim 0.4$ of their combined Einstein ring radius are detectable assuming a detection threshold of 3\%. For two $\mathbf{M}$ dwarfs, this corresponds to a limiting separation of $\gtrsim 1$ AU. Since very few observed $M$ dwarfs have companions at separations $\lesssim 1 \mathrm{AU}$, we conclude that close binaries will probably not corrupt the measurements of the mass function. We find that the detectability depends only weakly on the mass ratio. For those events for which individual masses can be determined, we find that binaries can be detected down to $b \sim 0.2$.
\end{abstract}

Subject headings: binaries: visual — gravitational lensing — stars: luminosity function, mass function

\section{INTRODUCTION}

Four surveys are currently discovering microlensing events toward the Large Magellanic Cloud and the Galactic bulge (Alcock et al. 1997; Aubourg et al. 1995; Udalski et al. 1994; Alard 1996). While the initial goal of these surveys was to determine the fraction of the halo that is composed of massive compact halo objects, the possible returns from these surveys are much broader. In particular, it may soon be possible to measure the mass function of the lenses.

Traditional methods of measuring the stellar mass function are restricted to luminous objects. Thus, these methods can be applied only to stars above the hydrogen-burning limit and are restricted to sparse samples near this limit. Microlensing overcomes this limitation because the effect is due to the mass of the lens, not its intrinsic luminosity. Thus, microlensing samples can extend mass function measurements beyond the hydrogen-burning limit.

In general, it is not possible to measure the masses of individual microlenses. This is because the only parameter that yields any information about the lens is the timescale, $t_{e}$, given by

$$
t_{e}=\frac{r_{e}}{v},
$$

where $v$ is the transverse velocity of the lens relative to the observer-source line of sight and $r_{e}$ is the Einstein ring radius,

$$
r_{e}^{2}=\frac{4 G M}{c^{2}} D_{\mathrm{OS}} z(1-z), \quad z=\frac{D_{\mathrm{OL}}}{D_{\mathrm{OS}}} .
$$

Here $D_{\mathrm{OL}}, D_{\mathrm{OS}}$, and $D_{\mathrm{LS}}$ are the distances between the observer, lens, and source and $M$ is the mass of the lens. Thus, $t_{e}$ is a complicated function of the quantities of interest: the mass, velocity, and distance of the lens. There are two basic methods of acquiring additional information. The first is

\footnotetext{
${ }^{1}$ Alfred P. Sloan Foundation Fellow.
}

using parallax to measure the projected Einstein radius of the lens, $\tilde{r}_{e}=\left(D_{\mathrm{OS}} / D_{\mathrm{LS}}\right) r_{e}$, either by considering the parallax caused by the motion of the Earth (Gould 1992; Alcock et al. 1995; Buchalter \& Kamionkowski 1997) or by employing a parallax satellite (Refsdal 1966; Gould 1995a; Boutreux \& Gould 1996; Gaudi \& Gould 1997). The second method is using proper motion information to measure the angular Einstein radius, $\theta_{e}=4 G M / c^{2} \tilde{r}_{e}$ (Gould 1994; Nemiroff \& Wickramasinghe 1994; Witt \& Mao 1994; Gould \& Welch 1996). Combining these two pieces of information yields the mass, distance, and velocity of the lens (Gould 1996 and references therein). Gould (1995b) estimates that one can expect $\sim 100$ giant events toward the Galactic bulge per year. A parallax satellite would be able to measure parallaxes for $\sim 70 \%$ of these events (Gaudi \& Gould 1997), and $\sim 15 \%$ of events could yield proper motions with current technology (Gould 1996). Thus, one might expect to obtain full information for $\sim 15$ events per year.

The fundamental limitation of using microlensing to reconstruct the mass function comes from the issue of unresolved binaries. If one assumes that the individual masses measured are due to single lenses without considering unresolved binaries, the reconstructed mass function will be biased toward large masses. This issue has been studied for the stellar mass function as determined from counts of luminous stars in the solar neighborhood (Reid 1991; Kroupa, Tout, \& Gilmore 1991), and it has been shown that unresolved binaries can significantly alter the inferred mass function, in particular, leading to an underestimation of the number of low-mass stars. Unresolved binaries could pose a similar problem for microlensing. It is thus important to quantify the detectability of binaries from microlensing.

Binary events can be divided into three basic classes according to the separation, $b$, in units of the Einstein ring radius: wide binaries $(b \gg 1)$, intermediate binaries $(b \sim 1)$, and close binaries $(b \ll 1)$. Although it may be difficult to determine the frequency of wide binaries from microlensing experiments, these objects pose no problem for reconstructing the mass function because the light curve for each 
member is unaffected by the presence of the other. Similarly, intermediate binaries pose no difficulty because they give rise to events that deviate dramatically from those of single lenses and, hence, are easily distinguished. However, close binaries are problematic in that they can masquerade as point lenses. In this way, microlensing differs from traditional methods of detecting binaries: the closer a companion is to a luminous star, the larger the induced orbital motion and hence the easier it is to detect spectroscopically.

The goal of this paper is to quantify the fraction of binary microlensing events for which the binarity of the lens is detectable. We specifically focus on close binaries, for which $b \leq 1$. In particular, we quantify the smallest separation that can be resolved for a majority of events given specified observing parameters. We also discuss the effects of the mass ratio of the binary components and effects of finite source size on the detectability.

\section{OBSERVED FREQUENCIES OF CLOSE BINARIES}

Before calculating detection rates, we first review what is known about the frequency of close binaries, specifically for low-mass stars and brown dwarfs, where microlensing is most useful. Unfortunately, there is no information about the binarity of objects with masses below the hydrogenburning limit, the regime of greatest interest. The most relevant observed sample is of the stars just above the hydrogen-burning limit. Various surveys of local, late-type dwarf stars have been made with the aim of discovering unseen companions. Precise radial velocity measurements are the most sensitive to low-mass, close binaries. Marcy \& Benitz (1989) obtained radial velocity measurements of $\mathbf{M}$ dwarfs in the solar neighborhood, with precisions of $\sim 200$ $\mathrm{m} \mathrm{s}{ }^{-1}$, allowing detection of companions with masses $\gtrsim 0.01 M_{\odot}$. Fischer \& Marcy (1992) examined this sample and found that out of 62 primaries, only three have a companion with separations of $\lesssim 1 \mathrm{AU}$. They estimate a detection probability of $86 \%$ for this range. For two $\mathrm{M}$ dwarfs, 1 AU corresponds to a separation in units of the Einstein ring radius of $b \sim 0.4$. Thus, approximately $3 /(0.86 \times 62) \sim 6 \%$ of $\mathbf{M}$ dwarfs in the solar neighborhood have companions with $b<0.4$. Although the solar neighborhood may not be a perfectly representative sample, it appears that binaries with separations $b<0.4$ are not common. As we show below, with observations of reasonable photometric precision, almost all binaries with separations of $b>0.4$ are detectable. Moreover, we show that for events where the mass can actually be measured, binarity is detectable even at substantially smaller separations. Thus, close binaries are unlikely to be a major source of error for reconstructing the mass function via microlensing.

\section{BINARY LENSING FORMALISM}

Consider a binary lens system. The Einstein ring radius for the binary system is given by equation (1b), where $M$ now denotes the total mass of the binary. We will normalize all subsequent lengths in the lens plane to $r_{e}$ and all lengths in the source plane to $r_{e}\left(D_{\mathrm{OS}} / D_{\mathrm{OL}}\right)$ the Einstein ring radius projected onto the source plane. All subsequent masses are normalized to $M$. Using complex coordinates, we denote the position of the source with respect to the center of mass of the binary as $\zeta=\xi+i \eta$ and the position of the component masses $m_{1}$ and $m_{2}$ as $z_{1}$ and $z_{2}$. The image positions with respect to the center of mass, $z=x+i y$, are then given by (Witt 1990)

$$
\zeta=z+\frac{m_{1}}{\bar{z}_{1}-\bar{z}}+\frac{m_{2}}{\bar{z}_{2}-\bar{z}} .
$$

The magnification, $A_{i}$, of each image is given by the Jacobian of the transformation (3a), evaluated at the image position,

$$
A_{i}=\left.\frac{1}{|\operatorname{det} J|}\right|_{z=z_{i}}, \quad \operatorname{det} J=1-\frac{\partial \zeta}{\partial \bar{z}} \frac{\overline{\partial \zeta}}{\partial \bar{z}} .
$$

If the images are unresolved, the total magnification is given by the sum of the individual magnifications, $A=\sum A_{i}$. The set of source positions where the magnification is formally infinite, given by the condition det $J=0$, defines closed curves called caustics. Five images are created if the source is inside a caustic, three if the source is outside. It is those regions near the caustics where the magnification from the binary deviates most dramatically from that of a point lens.

In practice, equation (3a) is solved numerically in order to determine the image positions, and these positions, together with equation (3b), are then used to calculate the total magnification.

\section{DETECTION RATES}

After normalizing to $r_{e}$ and $M$, there are two parameters that determine the lensing structure of a binary: $b$, the binary separation, and the mass ratio, $q=m_{1} / m_{2}$. To analyze how the magnification of a binary lens deviates from that of a point mass lens for a specified $q$ and $b$, we define $\epsilon$, the excess magnification over a single lens,

$$
\epsilon=\frac{A-A_{0}}{A_{0}}
$$

where $A_{0}$ is the magnification of a point lens with mass equal to the total mass of the binary, $M$, and located at the center of mass of the binary,

$$
A_{0}=\frac{|\zeta|^{2}+2}{|\zeta|\left(|\zeta|^{2}+4\right)^{1 / 2}} .
$$

We then calculate $\epsilon$ as a function of the source position, $(\xi, \eta)$ and draw contours of $\epsilon= \pm 3 \%, \pm 10 \%$. These excess magnification contours define regions where the magnification of the binary lens deviates from that of the single lens by $3 \%$ and $10 \%$. Figure 1 shows contours of $\epsilon$ for $q=0.1$, $0.4,0.7,1.0$ and $b=0.2,0.3,0.4,1.0$.

Ignoring higher-order effects (unresolved light from unmagnified sources, finite source, etc.), there are six parameters that characterize a binary lens event. Two of these are intrinsic to the lens: $b$ and $q$. Three are purely geometrical factors that describe the lens trajectory: $t_{0}$, the time of closest approach of the source and the center of mass of the lens; $\beta$, the separation between the source and center of mass at $t_{0}$; and $\theta$, the angle that the lens trajectory makes with the projected binary axis. The final parameter is the timescale of the event, given by equation (1a). A lensing event will be a straight line through the maps of $\epsilon$, specified by these six parameters. We want to know, given any event being observed with a specified sampling rate, $n_{\text {meas }}$, what the probability is of distinguishing a binary with parameters $q$ and $b$ from a point-mass lens. This means we must consider all possible $\beta$ and $\theta$. Since $t_{0}$ has no effect on the detection probability, we ignore it. For each possible trajec- 


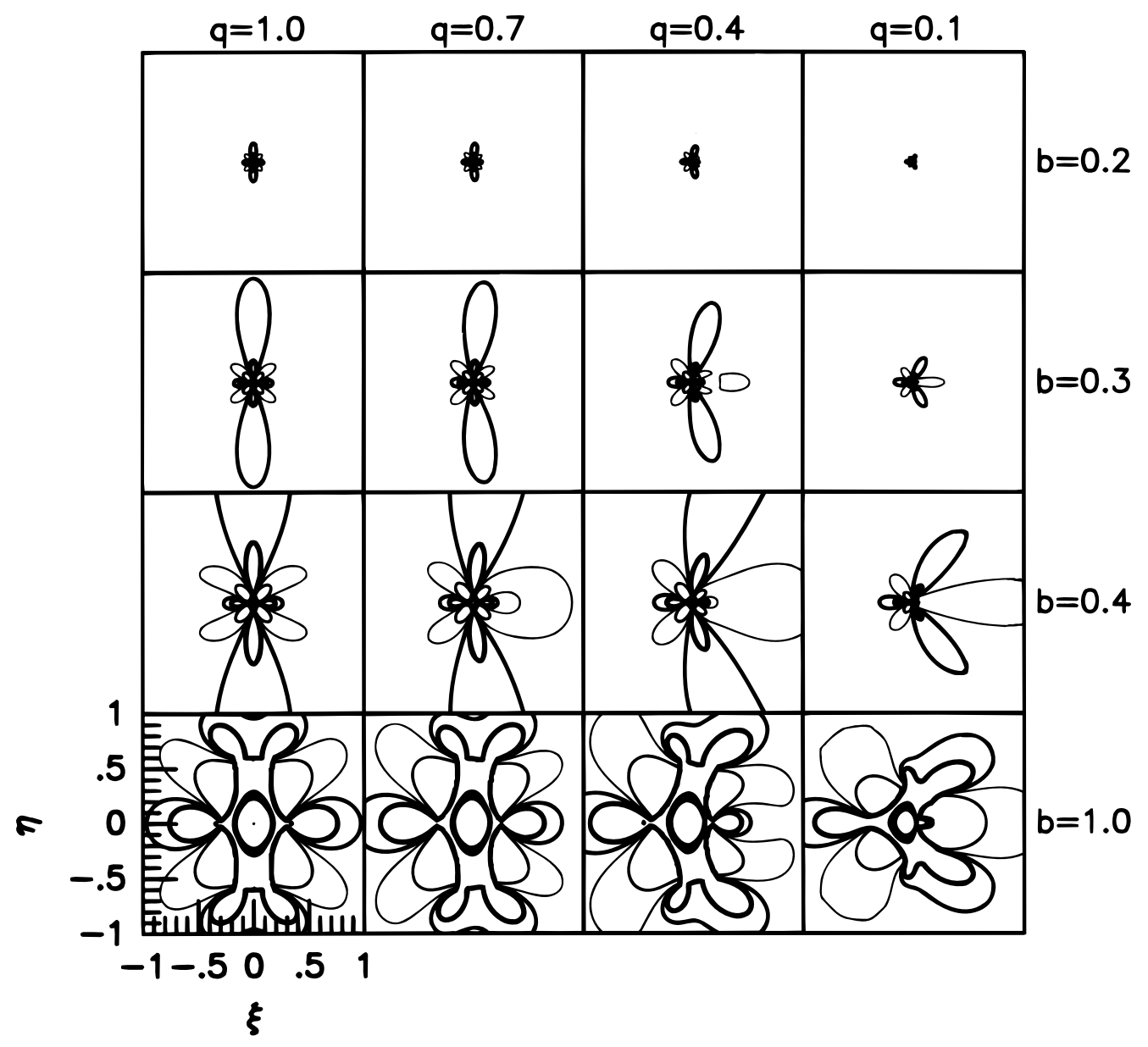

FIG. 1.-Contours of excess magnification, $\epsilon$, for four values of $b$, the binary separation in units of the Einstein ring, and $q$, the ratio of the masses of the binary components. Contours are $\epsilon=0.10$ (heaviest lines), $0.03,-0.10$, and -0.03 (lightest lines). The mass positions $z_{1}$ and $z_{2}$ are chosen such that the center of mass is at the origin, both masses are on the $\xi$-axis, and the smaller mass, $m_{1}$, is to the left.

tory, we ask whether, at each measurement, $|\epsilon| \geq \epsilon_{\text {thres }}$, where $\epsilon_{\text {thres }}$ is the given detection threshold. If this requirement is met, we consider that the binary has been detected. The distributions of $\beta$ and $\theta$ are flat. We therefore integrate over all trajectories with $0 \leq \beta \leq 1$ and $0 \leq \theta \leq 2 \pi$. The probability for detecting the binary is simply the ratio of the number of events for which the binary was detected to the total number of trial events. Comparing the panels of Figure 1, it is apparent that the binary detection probability depends much more strongly on $b$ than on $q$. For $b=1.0$, nearly all possible event trajectories will cross contours of $3 \%$, and therefore the fraction of events for which the binary is detected is $\sim 1$, whereas for $b=0.2$, the fraction of events for which the binary is detected is $\ll 1$. For $b=0.4$, a significant fraction of trajectories will still cross contours of $3 \%$. This implies that the binary detection probability must decline rapidly from $b=0.4$ to $b=0.2$.

In order to calculate binary detection probabilities, we adopt parameter values $\epsilon_{\text {thres }}=3 \%$ and $10 \%$ and $n_{\text {meas }}=$ 25 measurements per $r_{e}$. For $D_{\mathrm{OS}} \sim 8 \mathrm{kpc}$ and $D_{\mathrm{OL}} \sim 4 \mathrm{kpc}$,

$$
r_{e} \sim 4 \mathrm{AU}\left(\frac{M}{M_{\odot}}\right)^{1 / 2}
$$

Thus, for a binary consisting of two $\mathrm{M}$ stars, $M \sim 0.4 M_{\odot}$, $r_{e} \sim 3$ AU. Assuming $v \sim 200 \mathrm{~km} \mathrm{~s}^{-1}$, then $t_{e} \sim 25$ days. Therefore $n_{\text {meas }}$ corresponds to one measurement per day. Current follow-up surveys have temporal resolutions con- siderably greater than this; the PLANET collaboration reports a median sampling of $\sim 2 \mathrm{hr}$ in the I band (Albrow et al. 1996). Furthermore, from Figure 1 , for $b \geq 0.3$, the regions of $\epsilon>0.1$ are all larger than $\sim 0.1 r_{e}$, implying that deviations of less than $10 \%$ will persist for at last $\sim 2$ days. Therefore, each point on which we apply our detection criteria can be thought of as a bin of 12 independent PLANET measurements, and our detection criteria can be thought of as the requirement that 12 consecutive points have an average deviation of $\epsilon>\epsilon_{\text {thres }}$.

Figure 2 shows contours of binary detection probability as a function of $b$ and $q$ for $\epsilon_{\text {thres }}=10 \%$. As expected, $P$ is much more sensitive to $b$ than to $q$ and declines rapidly for $b \lesssim 0.5$. Excess magnification contours of $\epsilon=0.10$ follow closely the structure of the caustics. Thus, we can examine the structure of the caustics to understand Figure 2. In Figure 3, we show the caustics for three binary separations. For separations of $0.7 \lesssim b \leq 1.0$, there is only one caustic, and the binary detection probability is roughly given by the cross section of this caustic integrated over all angles $\theta$. At a binary separation of $b=2^{-1 / 2} \simeq 0.7$, the caustics splits into three parts. The main, diamond-shaped caustic is located near the center of mass, and the two smaller triangle-shaped caustics are on the $\eta$ axis symmetrically above and below the main caustic. As $b$ decreases from $b=0.7$, the secondary caustics become smaller and move farther from the center of mass, but the $\epsilon=0.1$ contour still extends between the 


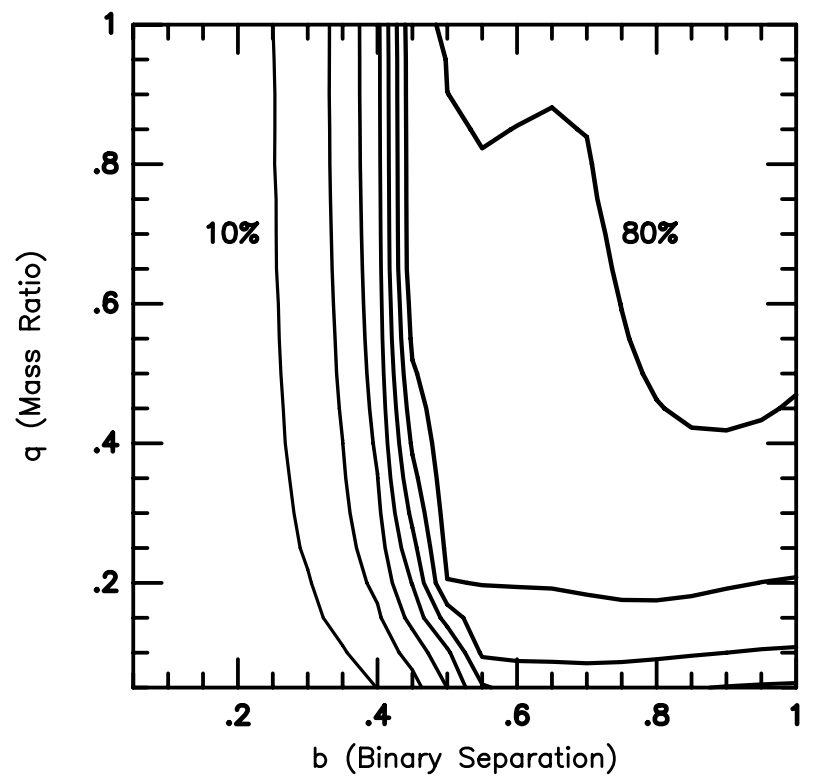

FIG. 2.-Contours of detection probability as a function of binary separation and mass ratio for $\epsilon_{\text {thres }}=0.10$. Contours have equal spacings of $10 \%$. A binary is considered detected if $|\epsilon|>0.10$ at any point during the event.

primary and secondary caustics. At $b \sim 0.5$, the $\epsilon=0.1$ contour breaks and no longer extends between the primary and secondary caustics. Thus, the binary detection probability shown in Figure 2 exhibits a sharp break at $b \sim 0.5$ and declines rapidly for $b<0.5$, as the primary caustic shrinks.

Figure 4 shows contours of binary detection probability as a function of $b$ and $q$ for $\epsilon_{\text {thres }}=3 \%$. Again, $P$ is much more sensitive to $b$ than to $q$ and declines rapidly for

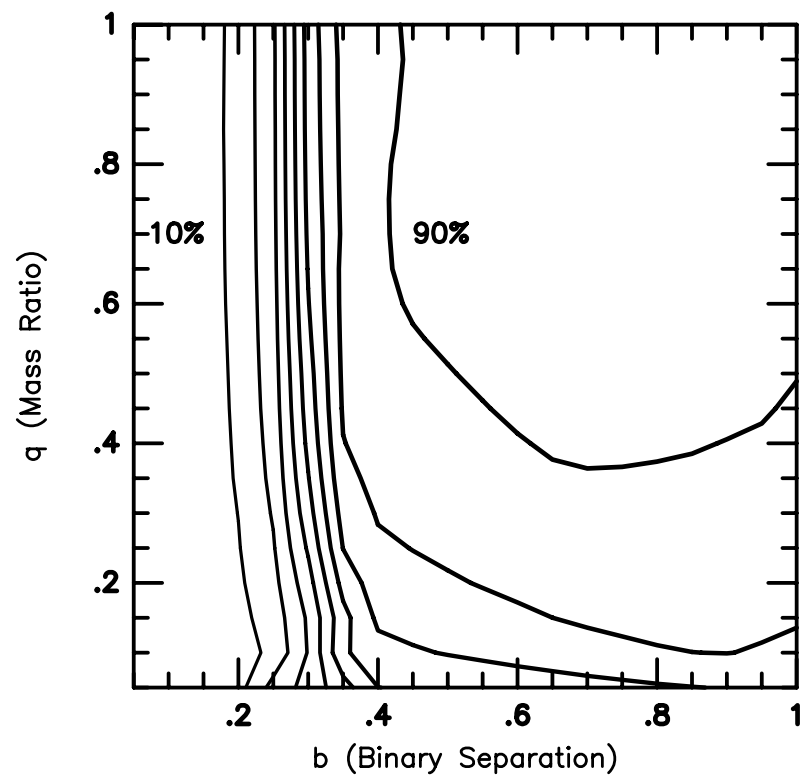

FIG. 4.-Contours of detection probability as a function of binary separation and mass ratio for $\epsilon_{\text {thres }}=0.03$. Contours have equal spacings of $10 \%$. A binary is considered detected if $|\epsilon|>0.03$ at any point during the event.

$b<0.4$. The reasons for the structure of Figure 4 are similar to those for $\epsilon_{\text {thres }}=3 \%$, but note that the $\epsilon=0.03$ contours follow the structure of the caustics less closely, and thus the break in the detection probability occurs at a smaller value of $b$.

\section{ORBITAL MOTIONS}

To understand the effect of the orbital motion of the binary on the detection probability, we define a parameter

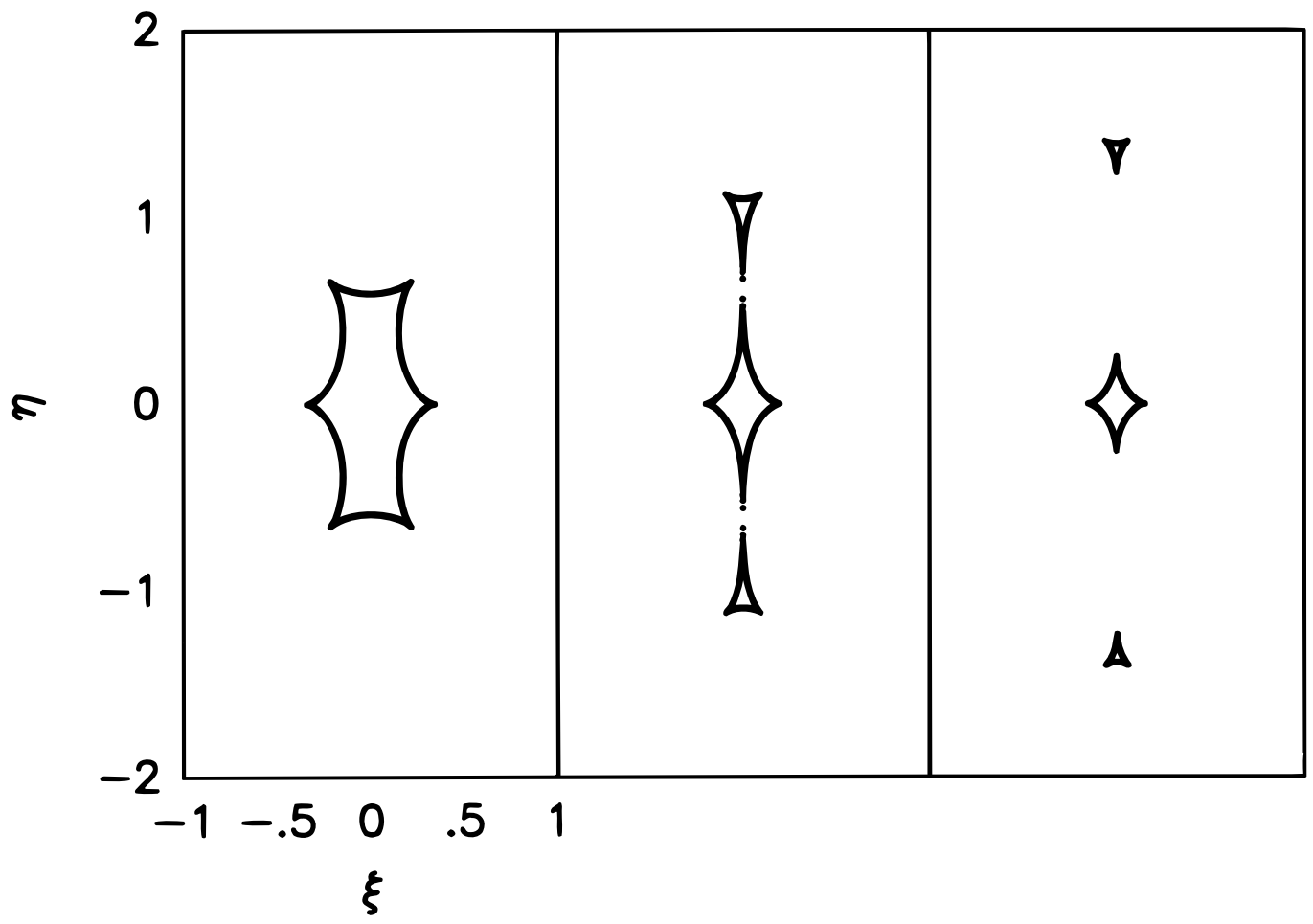

FIG. 3. Caustic curves, defined as the locus of points in the source plane where the magnification is formally infinite, for binary separations of $b=1.0$ (left), $2^{-1 / 2}$ (middle), 0.6 (right). 


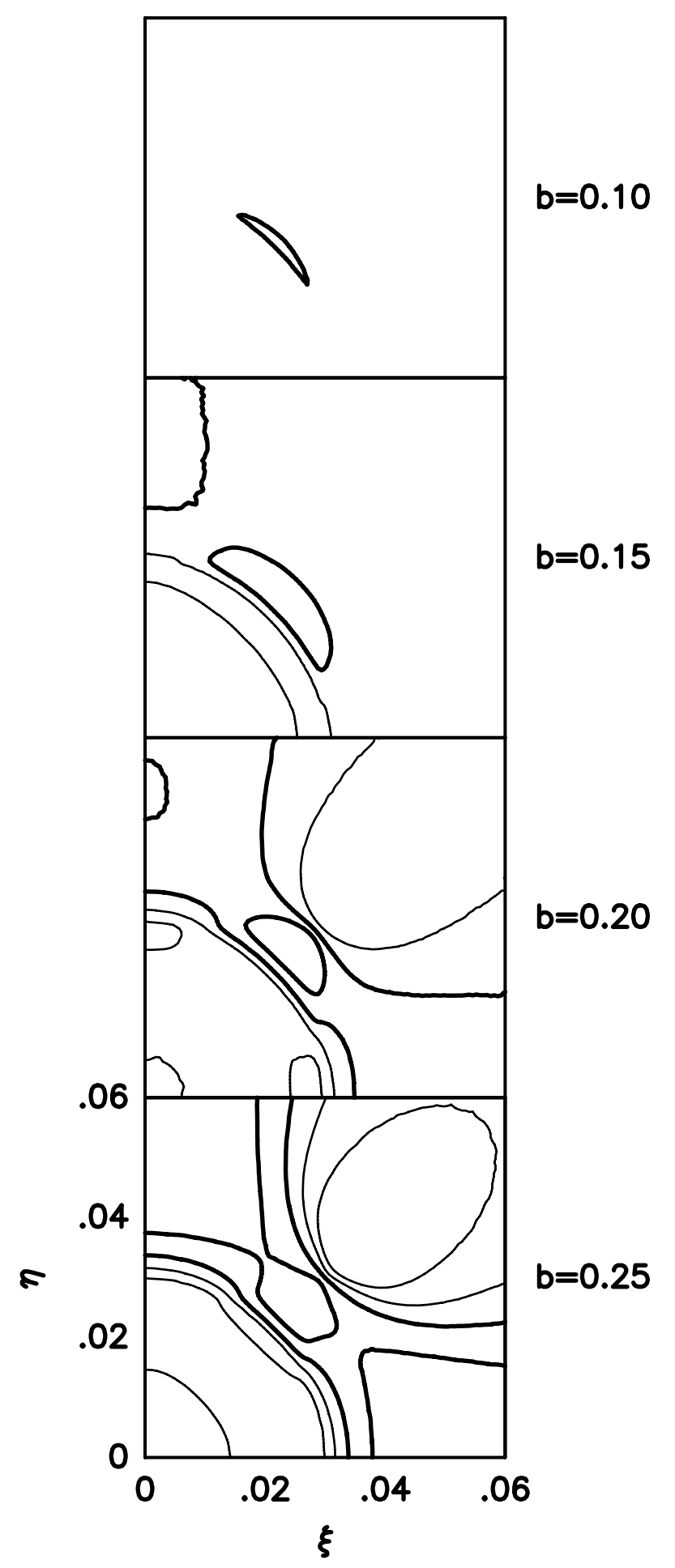

FIG. 5.-Contours of excess magnification, $\epsilon= \pm 0.03, \pm 0.10$, for a source of radius $\rho=0.03$ for events with $|\zeta| \leq 0.06$ for binary separations of $b=0.25,0.20,0.15,0.10$. Positive contours are bold curves.

$\psi$, which describes the amount the binary rotates during the event,

$$
\psi=2 \pi \frac{t_{e}}{P},
$$

where $P$ is the period of the binary. Using Kepler's laws, and assuming face-on circular orbits and $D_{\mathrm{Os}}=8 \mathrm{kpc}, \psi$

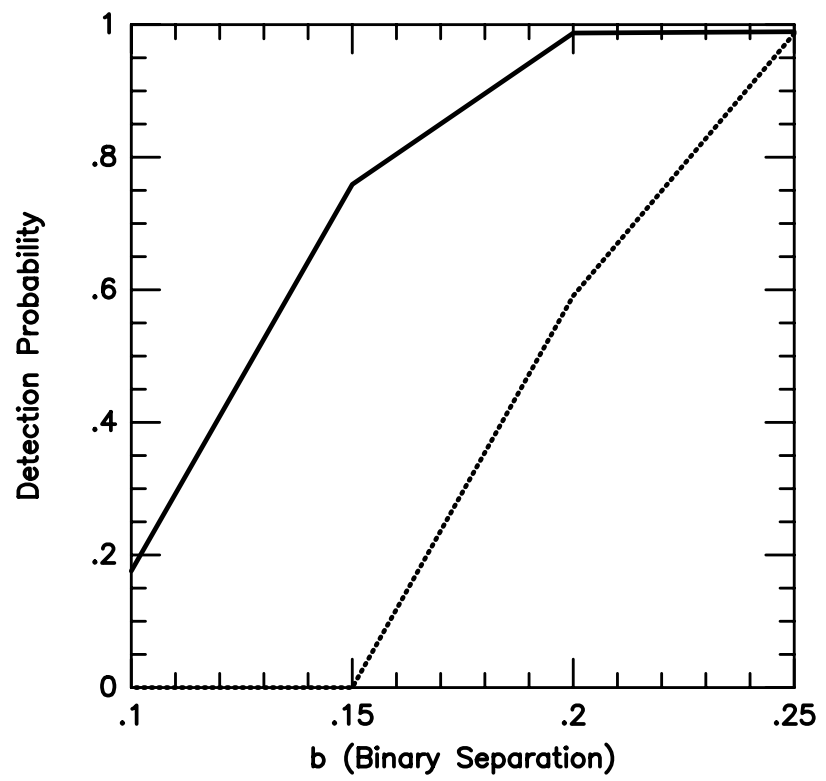

FIG. 6.-Detection probability as a function of binary separation for $\epsilon_{\text {thres }}=0.03$ (solid line), 0.10 (dotted line). The source size is $\rho=0.03$. A binary is considered detected if $|\epsilon|>\epsilon_{\text {thres }}$ at any point during the event.

can be written as

$$
\psi=\left(\frac{15 \mathrm{~km} \mathrm{~s}^{-1}}{v}\right)\left(\frac{M}{M_{\odot}}\right)^{1 / 4}[4 z(1-z)]^{1 / 4} b^{-3 / 2} .
$$

For $v \sim 200 \mathrm{~km} \mathrm{~s}^{-1}, z=\frac{1}{2}$, and $M \sim 0.4 M_{\odot}$, this becomes $\psi \sim 0.06 b^{3 / 2}$. Therefore, for $b>0.3$, the binary will rotate by $\psi \lesssim 20^{\circ}$. Thus, the rotation of the binary during an event is small for most events and will not change significantly the binary detection probabilities. This result is borne out quantitatively in numerical simulations that we have performed but which we do not report in detail.

\section{FINITE SOURCE EFFECTS}

In order to utilize microlensing as a method to reconstruct a mass function, one must be able to gather additional information for each individual event. One of the two necessary pieces of information is the proper motion of the lens, $\mu=v / D_{\mathrm{OL}}$. For events with relatively small Einstein rings (which are typically associated with the low-mass lenses considered here), $\mu$ can be measured primarily when the source passes very close to the lens. The light curve then deviates from that of a point source, and this deviation can then be used to determine $\mu$.

It is therefore interesting to restrict consideration to those events for which finite source effects must be taken into account and to ask what the probability is of detecting a binary if these effects are present. Since the majority of source stars for these events will be giants, we consider a source of average giant radius $R=22 R_{\odot}$ (Gould 1995b). For $D_{\mathrm{OS}}=8 \mathrm{kpc}, D_{\mathrm{OL}}=4 \mathrm{kpc}$, and $M \sim 0.4 M_{\odot}$, this corresponds to a projected distance on the source plane, normalized to $r_{e}$, of $\rho=0.03$. Gould \& Welch (1996) estimate that, using optical/infrared photometry, proper motions could be measured when $\beta \lesssim 2 \rho$. We therefore restrict our attention to those trajectories for which $\beta \leq 0.06$. In general, when a source crosses a caustic, the magnification will deviate dramatically from that of a point-mass lens, and the binary will be easily detectable. However, if the size of the caustic, 
which we will denote as $w$, is smaller than that of the source,

$$
w \lesssim \rho,
$$

then finite source effects will mask the binary magnification signature. If the caustic is very much smaller than the source, $w \ll \rho$, then the light curves for the binary and point-mass lenses will appear nearly identical when finite source effects are included. For $b \leq 0.3$, the primary caustic is diamond-shaped, and it can be shown analytically that

$$
w \simeq b^{2} / 2 .
$$

Combining equations (6a) and (6b), we find that finite source effects will partially mask the binary for $b \lesssim 0.25$.

To be more quantitative, we again calculate the excess magnification, $\epsilon$, over a point lens (cf. eq. [4.1]), where now $A$ and $A_{0}$ are the magnifications of a finite source of radius $\rho=0.03$. In order to calculate this magnification, we must integrate over the source. This could, in principle, be done directly in the source plane but would prove difficult because the magnification diverges as the source position approaches the caustic. We therefore employ the method suggested by Bennett \& Rhie (1996), of integrating in the image plane, where the magnification is well behaved. In this case, the magnification is simply given by

$$
A=\frac{\sum_{i=1}^{n} \Omega_{i}}{\Omega_{s}},
$$

where $\Omega_{i}$ is the area of image $i$ and $\Omega_{s}=\pi \rho^{2}$ is the area of the source. The difficulty, therefore, lies in finding the images, which in general are scattered throughout the image plane. Fortunately, for $b \leq 0.3$ and $|\zeta| \leq 0.06$, the images are all confined to a thin annulus of radius $|z|=1$ for both the binary and single lens. Using this method, we now construct contours of $\epsilon$ as a function of position in the source plane, $\zeta$, for a range of binary separations. In Figure 5 we show contours of $\epsilon= \pm 0.03$ and \pm 0.10 for $b=0.10,0.15$, $0.20,0.25$. Since the binary detection probability depends more strongly on $b$ than on $q$, we have included the results only for $q=1.0$. The results for other mass ratios are qualitatively similar.

As in $\S 3$, we calculate the binary detection probability by integrating over lens trajectories in the intervals $0 \leq \theta \leq 2 \pi$ and $0 \leq \beta \leq 1$. In Figure 6 we show the binary detection probability as a function of $b$ for the range $0.10 \leq b \leq 0.25$. Figure 6 shows that finite source effects decrease the detection probability for $b<0.25$ and render the binary virtually undetectable for $b \lesssim 0.1$, confirming the analytic estimate below equation (6a). Thus, although one could measure the proper motion of binary lenses with $b \lesssim 0.1$ (if $\beta \lesssim 0.6$ ), the binary nature of the lens would be unresolved, and one would attribute the event to a single lens. Therefore, when one attempted to reconstruct the mass function of the lenses using the proper motion information combined with parallax information (see $\S 1$ ), those binaries that were unresolved, namely, those with $b \lesssim 0.1$, would bias the reconstructed mass function toward larger masses. Fortunately, as discussed in $\S 2$, few luminous binaries are known with $b<0.4$. We therefore conclude that unresolved binaries are unlikely to be problematic in reconstructing the lens mass function for those events where proper motion information can be extracted.

\section{EXTREME MICROLENSING AND BINARY DETECTION}

There exists a small subclass of events in which it is possible to measure both the proper motion, $\mu$, and the projected Einstein radius, $\tilde{r}_{e}$, from ground-based measurement alone and thus determine the mass of the lens. These extreme microlensing events (EMEs) have been discussed by Gould (1997) and are characterized by a very high maximum magnification $A_{\max }$. The basic requirement to be able to measure $\mu$ and $\tilde{r}_{e}$ is $A_{\max } \gtrsim 200$ and

$$
\beta \lesssim \rho \text {. }
$$

We now determine whether it is possible to detect the presence of a binary of a given separation for these types of events. Gould (1996b) found that the typical source stars for EMEs are solar-type stars. For a source of physical radius $R \sim R_{\odot}$, the dimensionless radius is $\rho \simeq 0.001$ for $M=0.4$ $M_{\odot}, D_{\mathrm{OL}}=4 \mathrm{kpc}$, and $D_{\mathrm{OS}}=8 \mathrm{kpc}$. From equation (6a), we estimate that the binary will still be detectable as long as $w \geq 0.001$. Using the relation (6b), it is apparent that binaries of separation $b \gtrsim 0.05$ will be detectable in all EMEs. In fact, binaries of somewhat smaller separations will still be detectable, since as mentioned in $\S 6$, the requirement that $w \geq \rho$ is only approximate. Furthermore, to measure $r_{e}$, the sampling rate for EMEs must be very high, typically one observation per minute. These events typically have $I<17$; photon statistics predict photometric precisions of $\leq 1 \%$ for such an event with a 1 minute exposure assuming 1 " seeing, a $1 \mathrm{~m}$ telescope, and a sky brightness of $I=19.6 \mathrm{mag} \operatorname{arcsec}^{-2}$. With such high sampling rates and photometric precisions, one would be able to detect binaries of much smaller separations, perhaps down to $b \sim 0.01$.

We would like to thank P. Sackett for several stimulating discussions. This work was supported in part by grant AST 94-20746 from the NSF.

\section{REFERENCES}

Alard, C. 1996, in IAU Symp. 173, Astrophysical Applications of Gravitational Lensing, ed. C. S. Kochanek \& J. N. Hewitt (Dordrecht: Kluwer), 215

Albrow, M., et al. 1996, in IAU Symp. 173, Astrophysical Applications of Gravitational Lensing, ed. C. S. Kochanek \& J. N. Hewitt (Dordrecht: Kluwer), 227

Alcock, C., et al. 1995, ApJ, 454, L125

. 1997, ApJ, in press

Aubourg, E., et al. 1995, A\&A, 301,

Bennett, D., \& Rhie, S. 1996, ApJ, 472, 660

Boutreux, T., \& Gould, A. 1996, ApJ, 462, 705

Buchalter, A., \& Kamionkowski, M. 1997, ApJ, in press

Fischer, D., \& Marcy, G. 1992, ApJ, 396, 178

Gaudi, B. S., \& Gould, A. 1997, ApJ, 477, 152

Gould, A. 1992, ApJ, 392, 442
Gould, A. 1994, ApJ, 421, L71

1995a, ApJ, 441, L21

$1995 \mathrm{~b}, \mathrm{ApJ}, 447,491$

1996, PASP, 108,465

1997, ApJ, 480, 188

Gould, A., \& Welch, D. 1996, ApJ, 464, 212

Kroupa, P., Tout, C., \& Gilmore, G. 1991, MNRAS, 251, 293

Marcy, G., \& Benitz, K. 1989, ApJ, 344, 441

Nemiroff, R. J., \& Wickramasinghe, W. A. D. T. 1994, ApJ, 424, L21

Refsdal, S. 1966, MNRAS, 134, 315

Reid, N. 1991, AJ, 102, 1428

Udalski, A., et al. 1994, ApJ, 436, L103

Witt, H., \& Mao, S. 1994, ApJ, 430, 505

Witt, H. 1990, A\&A, 236, 311 